\title{
Aggregate Power Demand Model and Parameter Identification for Voltage Stability Enhancement
}

\author{
Jiansheng Huang , Zhuhan Jian, Leanne Rylands \\ School of Computing, Engineering and Mathematics, Western Sydney University, Australia
}

Copyright $(2016$ by authors, all rights reserved. Authors agree that this article remains permanently open access under the terms of the Creative Commons Attribution License 4.0 International License

\begin{abstract}
This paper focuses on the development of a fast aggregate electricity load model under steady-state and dynamic conditions. An important requirement of such a load model is that it should be robust, analytically sound, and conceptually clear. Besides, the model can be easily incorporated into existing programs of power system analysis. By using quasi-linear approximation made on the dynamic components of power demands, the general model is described in the paper by a combination of a linear system and a non-linear function. From the general model, the step response of the load is derived. The model is then reduced into a discrete one based on the step response. Successive applications of such a discrete model provide a good approximation of load dynamics. To evaluate parameters of the proposed discrete load model, an identification algorithm is developed to read data from a sliding sample window of bus voltages and load responses. By comparing the identification results with the detailed simulation of a test system, the proposed fast model is shown to provide good representation of the consumer load response under static and dynamic conditions. Furthermore, aggregate load characteristics have been taken into account in the voltage control of a study power system to demonstrate the importance of aggregate load modelling with respect to suppressing transients, reducing control errors and preventing reverse actions in voltage regulation.
\end{abstract}

Keywords Aggregate Power Demand, Discrete Dynamic Load Model, Associative Memory, Parameter Identification, Voltage Stability Enhancement

\section{Introduction}

Since load behaviors under voltage disturbances are dynamic, static load models are inadequate to apply in power system stability analysis and control $[1,2]$. Research was carried out in [3-5] to represent consumer loads by a collection of dynamic and steady load components. In [6] the step response of aggregate demand was derived. Due to nonlinear load characteristics, a single transfer function is obviously insufficient. It is more appropriate to use a combination of a linear system and nonlinear functions to describe power system dynamics and non-linear behavior during various phases of voltage disturbances [7-9].

This paper develops a fast consumer load model for the static and dynamic power demands. An important requirement on such a model is that the model has to be robust, analytically sound, and conceptually clear. The other important requirement include that the model can be easily employed in conventional programs of power flow and voltage stability analysis and control. Moreover, the model should be constructed with parameters that are often adopted to describe characteristics of aggregate power demands.

In the light of above requirements, we propose a discrete load model based on the step-response of power demands. Intuitively, such "discrete" model is attractive, since system and load controls are often carried out in discrete steps. We can save trouble of discretizing the load model in the applications. More importantly, the discrete load model can be applied recursively to provide an accurate representation of dynamic load response by representing continuous voltage variations with a series of small voltage steps and by assuming quasi-linearity of load dynamics during each step. The discrete load model is mathematically simple, and the solution times involved are short.

The paper is organized into six sections. Section 2 summarizes the general properties of an aggregate electricity load model and governing parameters. From the general first-order load model, Section 3 provides the justifications and derivation of the discrete load model. Developed algorithms are presented in Section 4 to identify the parameters determining the discrete load model. Section 5 compares the performance of the proposed load model and the parameter identification algorithm with the results generated from detailed simulation of a test system. Simulation is also performed on voltage control with and without considering the dynamic characteristics of the aggregate load demands. Section 6 contains the concluding remarks of this paper. 


\section{Parameters Governing Load Response}

A dynamic load model can be described with the differential equations in a general form:

$$
\begin{gathered}
f_{p}\left(P_{d}^{(n)}, P_{d}^{(n-1)}, \ldots, P_{d} ; v^{(n)}, v^{(n-1)}, \ldots, v\right)=0 \\
f_{Q}\left(Q_{d}^{(n)}, Q_{d}^{(n-1)}, \ldots, Q_{d} ; v^{(n)}, v^{(n-1)}, \ldots, v\right)=0
\end{gathered}
$$

where $v, P_{\mathrm{d}}$ and $Q_{\mathrm{d}}$ denote respectively the load voltage, the dynamic real and reactive power demands that are each time-varying.

If the voltage applied to the load is changed to $v$ and is then maintained constant during the transient period, both $P_{\mathrm{d}}$ and $Q_{\mathrm{d}}$ will settle down to the steady-state values $P_{\mathrm{s}}(v)$ and $Q_{\mathrm{s}}(v)$ as given by $[2,3]$ :

$$
\begin{gathered}
\left.P_{\mathrm{s}}(v)=P_{\mathrm{d}}\right] t \rightarrow \infty=c_{P} v^{\alpha} \\
\left.Q_{\mathrm{s}}(v)=Q_{\mathrm{d}}\right] t \rightarrow \infty=c_{Q} v^{\beta}
\end{gathered}
$$

where $c_{P} \& c_{\mathrm{Q}}$ are the coefficients denoting the steady-state power demands under unity load voltage, and the load type indices $\alpha \& \beta$ usually lie between $[0,2]$.

To show the manner in which the dynamic power demand approaches the static value after the step voltage change, new variables representing the dynamic load components, i.e., the difference between dynamic and static power demands are introduced. They are $u_{P}=P_{d}-P_{s}(v)$ and $u_{Q}=Q_{d}-Q_{s}(v)$ used in the paper to describe the dynamic performance of power system loads.

When a first-order load model is considered, the dynamic load is represented by a nonlinear differential equation of the form:

$$
T_{P} u_{P}+u_{P}=f(v, \dot{v})
$$

where $T_{\mathrm{P}}$ is the load time constant, and $f(v, v)$ is a function of voltage variations during the transient period. By adopting quasi-linear approximation, the $f(v, \dot{v})$ is further simplified into:

$$
f(v, \dot{v})=\lambda \dot{v}
$$

and Eqn. 3 becomes:

$$
T_{P} \dot{u}_{P}+u_{P}=\lambda \dot{v}
$$

The reactive power demand can be modeled in a similar way. The simplification in Eqn. 4 shows the load dynamics as inspired by voltage variations during transients. The coefficient $\lambda$ depends upon the system state immediately before the current point $\left(P_{\mathrm{d}}\left(t^{-}\right)\right.$and $\left.v\left(t^{-}\right)\right)$. Consequently the $\lambda$ should be updated continuously with $P_{\mathrm{d}}\left(t^{-}\right)$and $v\left(t^{-}\right)$that are the signals obtained from $P_{\mathrm{d}}$ and $v$ through a delay unit respectively. The load model as in Eqn. 5 is illustrated in Fig. 1 , where the two blocks " $d / d t$ " and " $h(t)$ " together with the coefficient " $\lambda$ " describe the load dynamics due to continuous voltage variations. The block $h(t)$ is the unforced solution of Eqn. 5. Obviously the proposed model is a recursive one (via $\lambda)$.

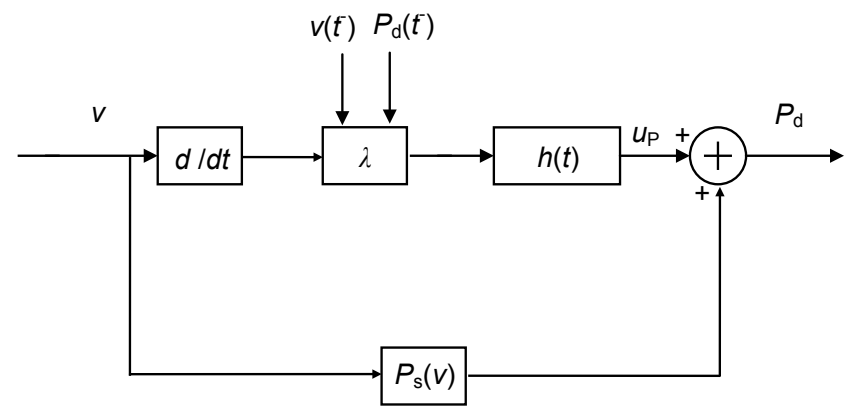

Figure 1. Diagram of the general dynamic load model

\section{Step Response and Discrete Dynamic Load Model}

Referring to the general load model (Eqn.3), if the voltage variation occurs only at the beginning of the transient period, the function $f(v, \dot{v})(=\lambda \dot{v})$ will be zero thereafter. The load behavior under such step voltage change, say from $v_{0}$ to $v_{1}$, can then be simulated by a change of the initial condition at $t=t_{0}$, with Eqn. 3 in a homogeneous form and the load model as a step response:

$$
T_{P} u_{P}+u_{P}=0
$$

whose solution is $u_{P}=\left[\frac{v_{1}^{2}}{v_{0}^{2}} P_{d}\left(t_{0}^{-}\right)-P_{s}\left(v_{1}\right)\right] e^{-\frac{t-t_{0}}{T_{P}}}$ and $P_{\mathrm{d}}$ is given by:

$$
P_{d}=\left[\frac{v_{1}^{2}}{v_{0}^{2}} P_{d}\left(t_{0}^{-}\right)-P_{s}\left(v_{1}\right)\right] e^{-\frac{t-t_{0}}{T_{P}}}+P_{s}\left(v_{1}\right), \quad \mathrm{t} \geq \mathrm{t} 0,
$$

where $c_{\mathrm{P}}, \alpha$ and $T_{\mathrm{P}}$ are the governing load parameters, $P_{\mathrm{d}}\left(t_{0}{ }^{-}\right)$ represents the power demand immediately before the voltage change. The Eqn. 7 describes the power demand variation with an initial voltage of $v_{0}$ and an applied voltage of $v_{1}$ at $t_{0}$. Before the voltage change, the load could be in steady state or dynamic state with the $P_{\mathrm{d}}\left(t_{0}{ }^{-}\right)$equal to or not equal to $P_{\mathrm{s}}\left(v_{0}\right)$. The Eqn.7 forms the basis of the discrete load model as described below.

From Eqn. 7, the power demand immediately after the step voltage change $\left(t=t_{0}{ }^{+}\right)$should be given by:

$$
P_{d}\left(t_{0}^{+}\right)=\frac{v_{1}^{2}}{v_{0}^{2}} P_{d}\left(t_{0}^{-}\right)
$$

Unlike the load model reported in [5], the above power jump depends on the initial conditions $v_{0}$ and $P_{\mathrm{d}}\left(t_{0}{ }^{-}\right)$, which agrees with the known facts.

Eqn. 7 simulates the load dynamics due to an initial step 
voltage change. During subsequent transients the load voltage is assumed constant. Thus the step response cannot be directly applied to describe the power demands under general load voltage. By breaking down a range of continuous voltage variations into small and equal time intervals $T_{\mathrm{s}}$ and assuming the applied voltage to be piece-wise constant in each interval, however, a sequence of dynamic power demand $P_{\mathrm{d}}\left(t_{0}+n T_{\mathrm{s}}\right)$ can then be used to represent the actual dynamic load response. In the following text and for simplification, $P_{\mathrm{d}}\left(t_{0}+n T_{\mathrm{s}}\right)$ is represented as $P_{\mathrm{d}}(n)$ (voltage samples have a similar representation: $v(n)$ or $v_{n}$ ), and the demands immediately before and after each $n^{\text {th }}$ voltage sampling point are respectively as $P_{\mathrm{d}}\left(n^{-}\right)$and $P_{\mathrm{d}}\left(n^{+}\right)$. According to Eqn. 7 we have:

$$
\begin{aligned}
& P_{d}\left(n^{-}\right)=P_{s}\left(v_{n-1}\right)+ \\
& {\left[P_{d}\left(n-1^{+}\right)-P_{s}\left(v_{n-1}\right)\right] \exp \left(-T_{s} / T_{P}\right)} \\
& \quad P_{d}\left(n^{+}\right)=\left(v_{n}^{2} / v_{n-1}^{2}\right) P_{d}\left(n^{-}\right)
\end{aligned}
$$

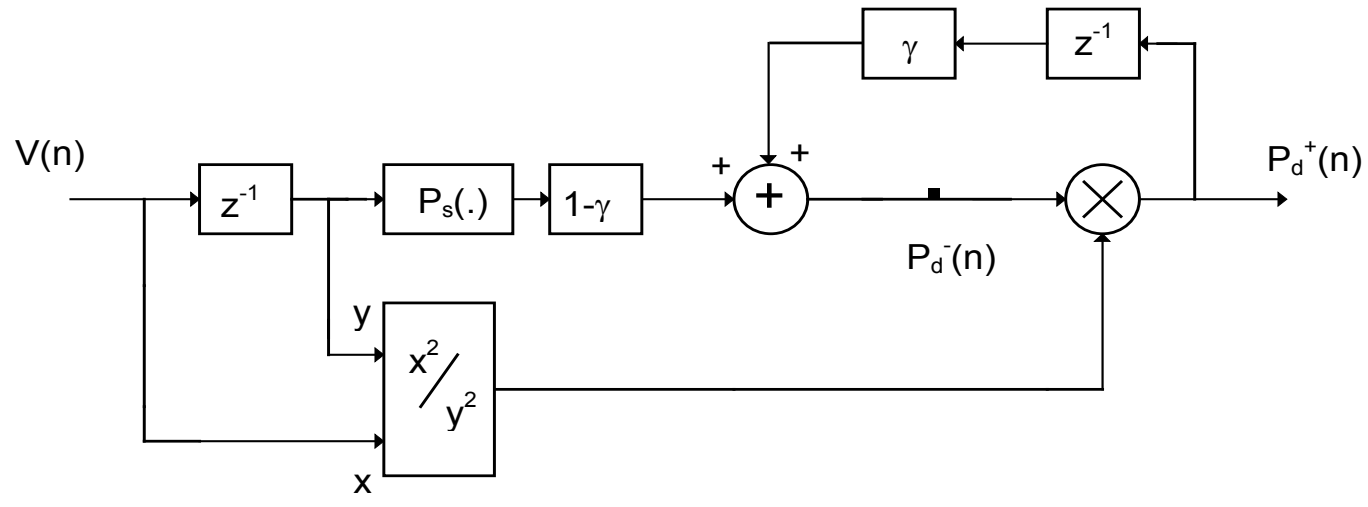

Figure 2. Diagram of the discrete Dynamic Load Model

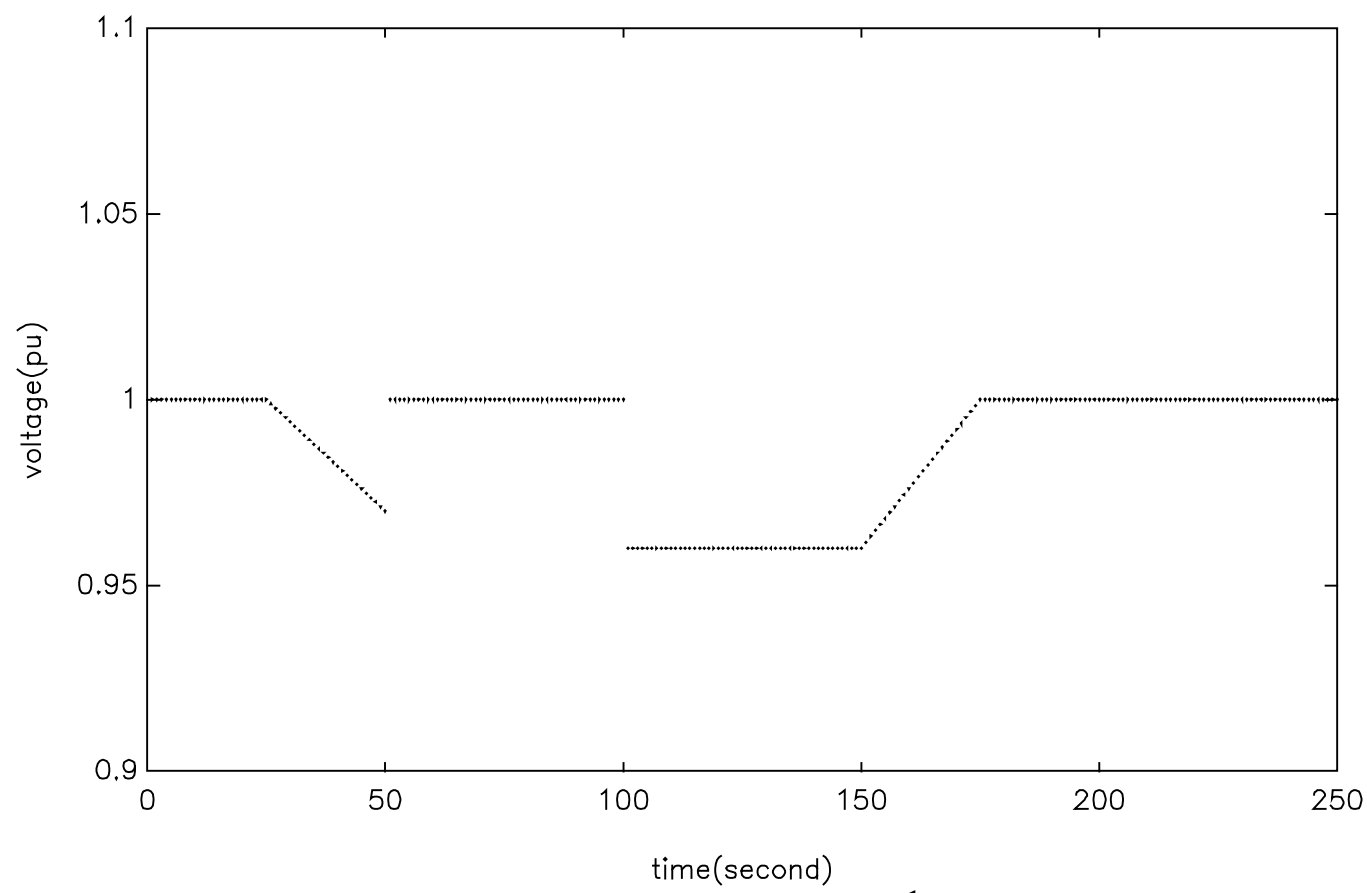

Figure 3a. Voltage sequence v(n), $\mathrm{T}_{\mathrm{s}}=1 s$
By expressing $P_{\mathrm{d}}\left(n^{-}\right)$and $P_{\mathrm{d}}\left(n^{+}\right)$respectively as $P_{\mathrm{d}}^{-}(n)$ and $P_{\mathrm{d}}^{+}(n)$, and rewriting $P_{\mathrm{d}}^{-}(n)$ as $P_{\mathrm{d}}^{-}(n)=(1-\gamma) P_{\mathrm{s}}\left(v_{\mathrm{n}-1}\right)+\gamma P_{\mathrm{d}}^{+}$ $(n-1)$ with $\gamma=\exp \left(-T_{\mathrm{s}} / T_{\mathrm{P}}\right)(0<\gamma<1)$, we generate $P_{\mathrm{d}}^{-}(n)$ and $P_{\mathrm{d}}^{+}(n)$ as shown in Fig. 2.

For a continuous voltage curve, if the sampling interval is sufficiently small $\left(T_{\mathrm{s}}<<T_{\mathrm{P}}\right), P_{\mathrm{d}}^{-}(n)$ and $P_{\mathrm{d}}^{+}(n)$ will be very close to each other, and either of them will be a good approximation of the power demand. Under the load voltage represented by a discrete sequence $v(n)$ as shown in Fig. 3a, taking for example, $P_{\mathrm{d}}^{-}(n)$ and $P_{\mathrm{d}}^{+}(n)$ (Fig. 3b) are obtained from the discrete model as illustrated in Fig.2. The actual power response is a piece-wise exponential curve with the two series $P_{\mathrm{d}}^{+}(n)$ and $P_{\mathrm{d}}^{-}(n+1)$ at each interval $\left[n T_{\mathrm{s}},(n+1) T_{\mathrm{s}}\right]$ being connected by the exponential curve. $P_{\mathrm{d}}^{-}(n)$ and $P_{\mathrm{d}}^{+}(n)$ are respectively the inner and outer envelopes of the actual power demand curve. Thus a sequence averaged from $P_{\mathrm{d}}^{-}(n)$ and $P_{\mathrm{d}}^{+}(n)$ series can also be used as an approximation without the discontinuities for the actual response. 


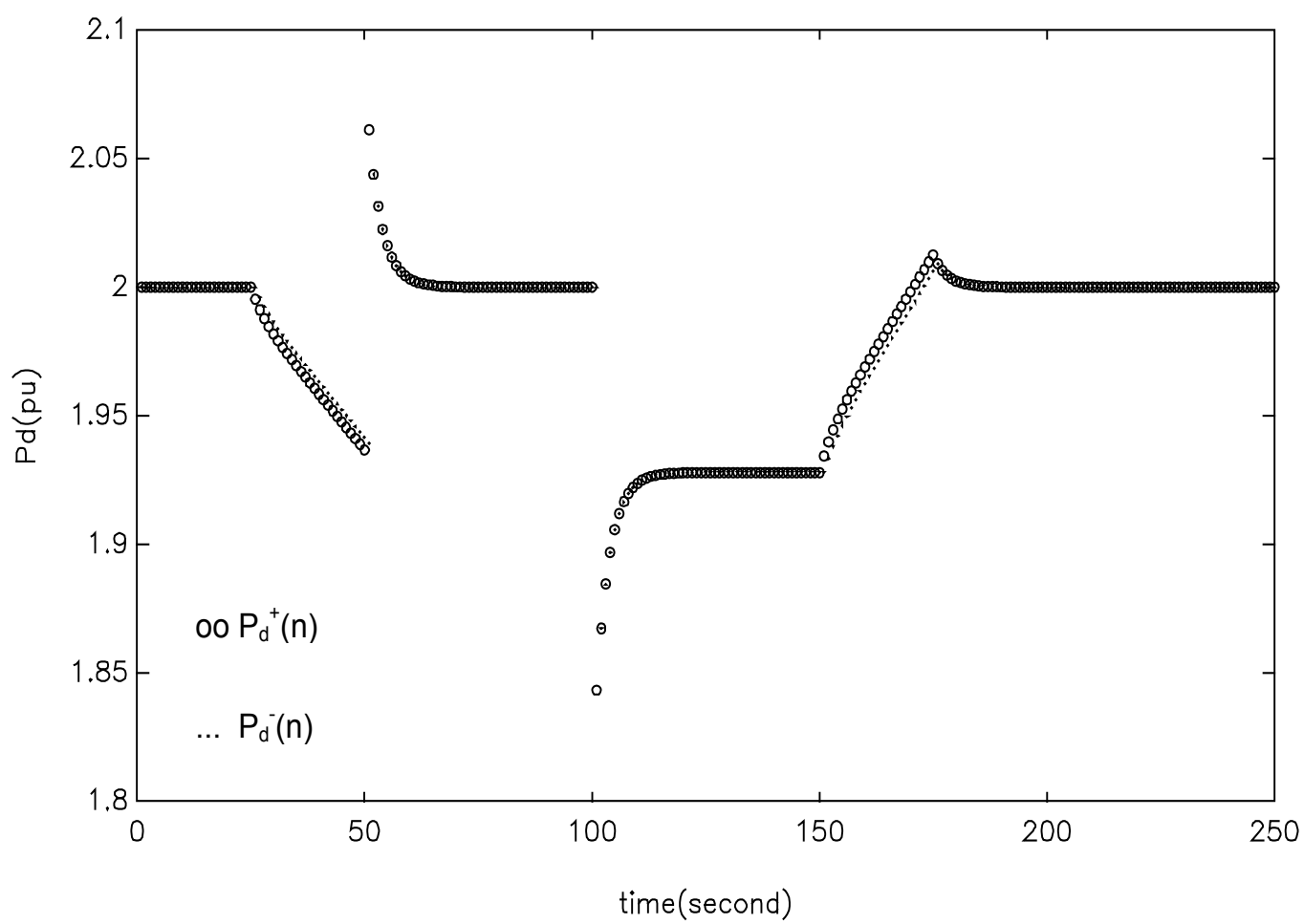

Figure 3b. Power demand before and after each voltage change $P_{\mathrm{d}}^{-}(n)$ and $P_{\mathrm{d}}^{+}(n) c_{\mathrm{P}}=2, \alpha=0.9, T_{\mathrm{P}}=3 s$

The continuous load model illustrated in Fig.1 is general enough to give an accurate description of the load in different states. In applications, the continuous model should be discretized by using, for example, the trapezoidal approach. As an alternative, we can use the developed discrete model. The model can be used directly, simpler and faster. By using the discrete load model and representing the continuous voltage variations in steps, interactions between the loads and the power system can be easily evaluated with conventional load flow and other voltage stability analysis programs. In addition, most of the voltage/reactive control actions are discrete and can be directly included. Load modeling is broken down into small and equal time intervals with $P_{\mathrm{d}}(n)$ being determined by $P_{\mathrm{d}}(n-1)$, and load voltages $v_{\mathrm{n}}$ and $v_{\mathrm{n}-1}$ within the same time interval: $\left(v_{n}^{2} / v_{n-1}^{2}\right) P_{d}(n-1)$ which can be applied in the iterations of conventional power flow programs. In general, as the load varies so does the bus voltage. Since the sampling and the power flow calculating can only be done in finite time points (no matter a continuous or a discrete model is used), an assumption of piece-wise constant load voltage is appropriate. The developed discrete load model possesses the advantages of analytically sound, conceptually clear, simple in structure, and fast in computation.

\section{Identification of Load Parameters}

The proposed discrete load model (Eqns. 9 and 10) is described by three load parameters $c_{\mathrm{P}}, \alpha$, and $T_{\mathrm{P}}$. A successive linearization techniques based algorithm is developed for identifying these parameters. In the algorithm, the set of observed vectors of voltages $\mathbf{V}\left(\left\{\mathrm{v}_{\mathrm{n}}\right\}\right)$ and power demands $\mathbf{P}\left(\left\{P_{\mathrm{d}}(n)\right\}\right)$ are grouped in training segments. The algorithm evaluates the load parameters (represented by the vector $\mathbf{L}_{\mathbf{P}}$ ) in each training segment of observed voltages and power demands. For every training segment, the function $\mathbf{L}_{\mathbf{P}}$ $=f(\mathbf{V}, \mathbf{P})$ is approximated by a linear neural network. Unlike nonlinear cases, the linear neural network can be constructed directly from the training set without iterations [13]:

$$
\mathbf{L}_{P}=\mathbf{W}\left[\begin{array}{l}
\mathbf{V} \\
\mathbf{P}
\end{array}\right]+\mathbf{B}
$$

where $\mathbf{W}$ is the memory matrix and $\mathbf{B}$ is the bias vector. For a training set consisting of $\left\{\mathbf{V}_{\mathrm{i}}, \mathbf{P}_{\mathrm{i}}\right\}$ and $\left\{\mathbf{L}_{\mathrm{p}}{ }^{\mathrm{i}}\right\}, i=1,2, \ldots, \mathrm{N}$, we seek $\mathbf{W}$ and $\mathbf{B}$ such that the error of the linear approximation is minimized. To prevent ill-conditioned calculation, a penalty factor is introduced. Thus a multi-criterion cost function is built up for the evaluation of $\mathbf{W}$ and $\mathbf{B}$ :

$$
J=(1-a)\|\mathbf{W}\|^{2}+a \sum_{i=1}^{N}\left\|W\left[\begin{array}{l}
V_{i} \\
P_{i}
\end{array}\right]+B-\mathbf{L}_{P}^{i}\right\|^{2}
$$

where $\|\cdot\|$ denotes the Euclidean norm and " $a$ " reflects the relative emphasis on the cost of the identification error and the cost of $\mathbf{W}$ being ill-conditioned. Minimizing $J$ by letting the derivative of $J$ with respect to $\mathbf{W}$ and $\mathbf{B}$ equal zero, we obtain:

$$
\tilde{\mathbf{W}}=a \mathbf{W}_{1} \mathbf{W}_{2}
$$




$$
\begin{gathered}
\mathbf{W}_{1}=\sum_{i=1}^{N}\left[\mathbf{L}_{P}^{i}\left[\begin{array}{l}
\mathbf{V}_{i} \\
\mathbf{P}_{i}
\end{array}\right]^{T}-\frac{1}{N} \sum_{j=1}^{N} \mathbf{L}_{P}^{j}\left[\begin{array}{l}
\mathbf{V}_{i} \\
\mathbf{P}_{i}
\end{array}\right]^{T}\right] \\
\mathbf{W}_{2}=\left[(1-a) \mathbf{I}+a \sum_{i=1}^{N}\left(\left[\begin{array}{l}
\mathbf{V}_{i} \\
\mathbf{P}_{i}
\end{array}\right]\left[\begin{array}{l}
\mathbf{V}_{i} \\
\mathbf{P}_{i}
\end{array}\right]^{T}-\frac{1}{N} \sum_{j=1}^{N}\left[\begin{array}{l}
V_{j} \\
P_{j}
\end{array}\right]\left[\begin{array}{c}
V_{j} \\
P_{j}
\end{array}\right]^{T}\right)\right]^{-1} \\
\tilde{\mathbf{B}}=\frac{1}{N} \sum_{i=1}^{N}\left(\mathbf{L}_{P}^{i}-\tilde{\mathbf{W}}\left[\begin{array}{c}
\mathbf{V}_{i} \\
\mathbf{P}_{i}
\end{array}\right]\right)
\end{gathered}
$$

Because the function $\mathbf{L}_{\mathbf{P}}=f(\mathbf{V}, \mathbf{P})$ is highly nonlinear, it is inappropriate to linearize $f(\mathbf{V}, \mathbf{P})$ in a wide range so as to get a reasonable estimate of $\mathbf{L}_{\mathbf{p}}$. Thus the linearization is only performed in a local vicinity of the current observed voltage segment $\mathbf{V}$ and the initial estimation of the load parameters $\mathbf{L}_{\mathbf{P}}$. With zero mean Gaussian noises added to data of $\mathbf{V}$ and $\mathbf{L}_{\mathbf{p}}, \mathbf{P}$ is predicted by solving a forward problem (with the discrete model). In this way the training set of the linear neural network $\left(\left\{\mathbf{V}_{\mathrm{i}}, \mathbf{P}_{\mathrm{i}}\right\}-\left\{\mathbf{L}_{\mathrm{p}}{ }^{\mathrm{i}}\right\}\right)$ is constructed. Obviously the load parameters identified from the current $\mathbf{V}$ and $\mathbf{P}$ can be used as the initial estimation for the next sample window. Estimation of load parameters is further improved as follows. For the identification of $\tilde{\mathbf{L}}_{P}=\tilde{\mathbf{W}}\left[\begin{array}{l}\mathbf{V} \\ \mathbf{P}\end{array}\right]+\tilde{\mathbf{B}}$, denote $\widetilde{\mathbf{P}}$ as the predicted power demand through solving the forward problem with $\tilde{\mathbf{L}}_{P}$ and $\mathbf{V}$. Accordingly $\mathbf{P}-\tilde{\mathbf{P}}$ is the discrepancy brought about by the error of the load parameter estimation. Next, suppose that an exact local linear expression of $\mathbf{L}_{\mathbf{P}}$ with respect to $\mathbf{V}$ and $\mathbf{P}$ is available with $\mathbf{W}^{*}$ and $\mathbf{B} *$ represent the exact expression and $\mathbf{L}_{\mathrm{P}} *$ is the true value of $\mathbf{L}_{P}$. A more accurate estimation of $\mathbf{L}_{P}$ is obtained as:

$$
\mathbf{L}_{P} *=\tilde{\mathbf{L}}_{P}+\mathbf{W} *\left[\begin{array}{c}
\mathbf{0} \\
\mathbf{P}-\tilde{\mathbf{P}}
\end{array}\right] \approx \tilde{\mathbf{L}}_{P}+\tilde{\mathbf{W}}\left[\begin{array}{c}
\mathbf{0} \\
\mathbf{P}-\tilde{\mathbf{P}}
\end{array}\right]
$$

by taking the approximation of $(\mathbf{W} *-\tilde{\mathbf{W}})\left[\begin{array}{c}\mathbf{0} \\ \mathbf{P}-\tilde{\mathbf{P}}\end{array}\right]=0[13]$.
Load modelling and parameter identification for reactive power demands can be performed in a similar way.

\section{Simulations}

Two case studies are carried out, with one for the load modelling and parameter identification and the other for voltage control with and without considering transient power demands.

\subsection{Load Modelling}

A constant resistor and an induction motor are connected in parallel to form the test system. The observed load response (Fig. 4b) is generated using detailed dynamic simulations for load voltage variations as shown in Fig. 4a. A constant load torque is assumed for the motor, while the rotational and core losses are combined into a constant load demand. Rotor flux dynamics are not considered since the associated time constants are usually very small. In addition, the supply frequency is assumed constant as in standard voltage stability analysis.

Using the successive linearization method, the only requirement to the training segment is that it contains sufficient variations of load voltage. Hence each sample window of the load voltage and the power can be chosen very short. By moving the sample window along the measured curves, the load parameters can be continuously identified as shown in Fig.5a. In the simulation, the initial estimation of the load parameters are given as $c_{\mathrm{P}}=1.3 \mathrm{pu}, \alpha$ $=1.0, T_{\mathrm{P}}=6 T_{\mathrm{s}}\left(T_{\mathrm{s}}=0.1 \mathrm{~s}\right)$. It is found that the estimation of $c_{\mathrm{P}}$ has a very fast convergence while the $\alpha$ and $T_{\mathrm{P}}$ have comparatively slower ones. The identified parameters from the last sample window are $c_{\mathrm{P}}=1.0000 \mathrm{pu}, \alpha=1.2479, T_{\mathrm{P}}=$ $6.3850 T_{\mathrm{s}}$. The prediction error for each sample window (use the parameters identified from each sample window to predict the power demand over the whole range and compare the predicted power with the actual one) are plotted in Fig. $5 \mathrm{~b}$. 


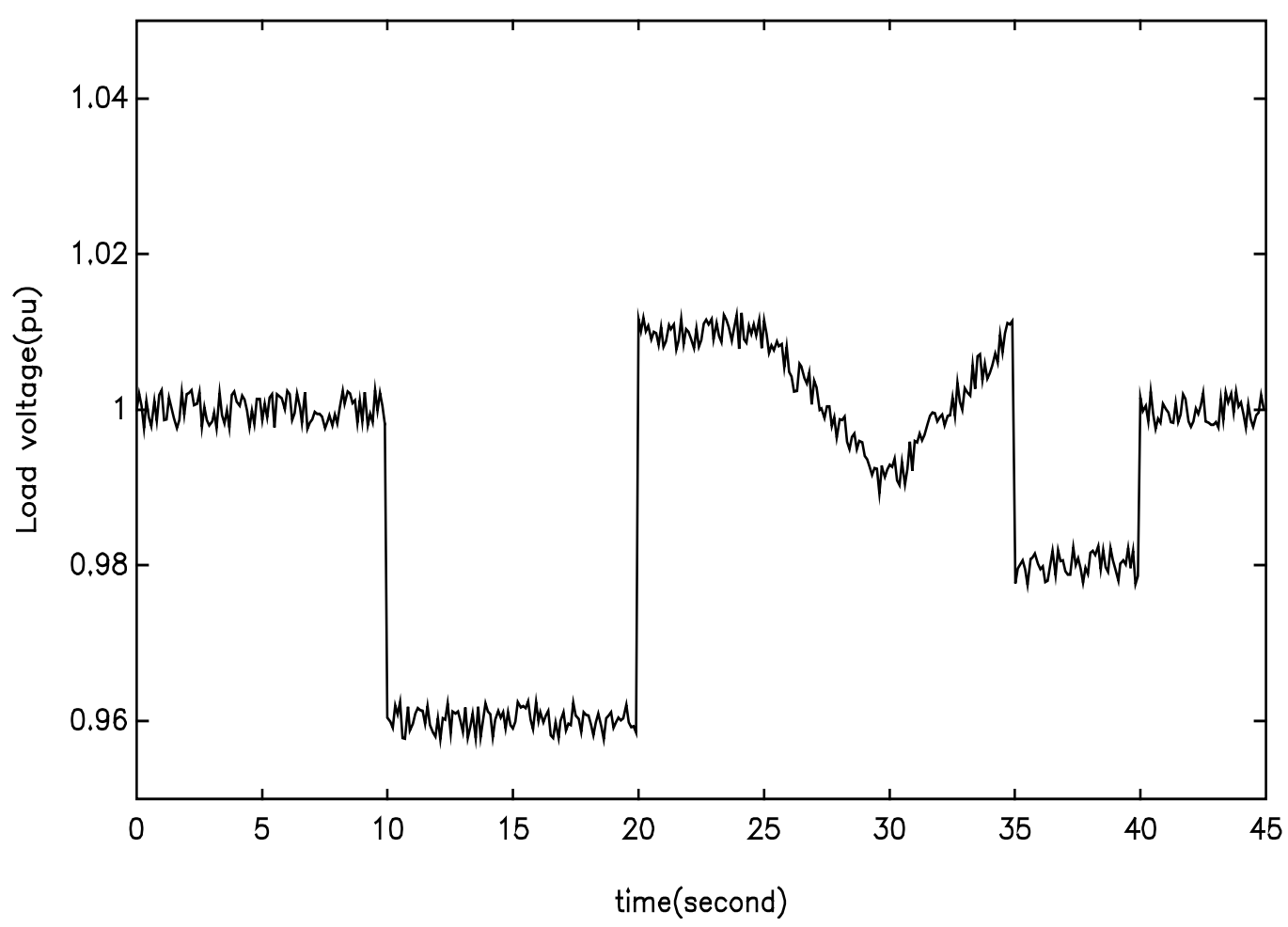

Figure 4a. Load voltage

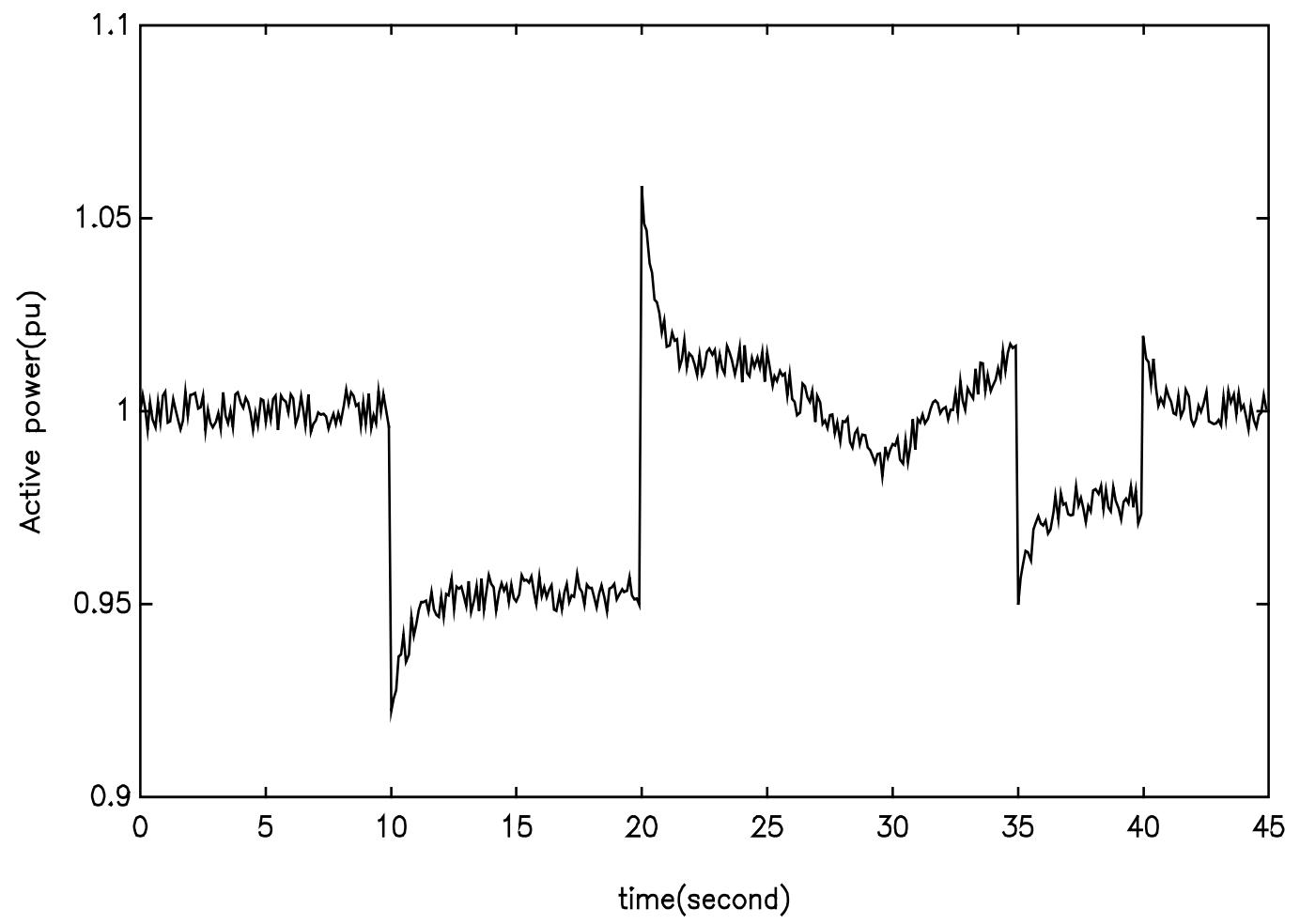

Figure 4b. "Observed' power demand 


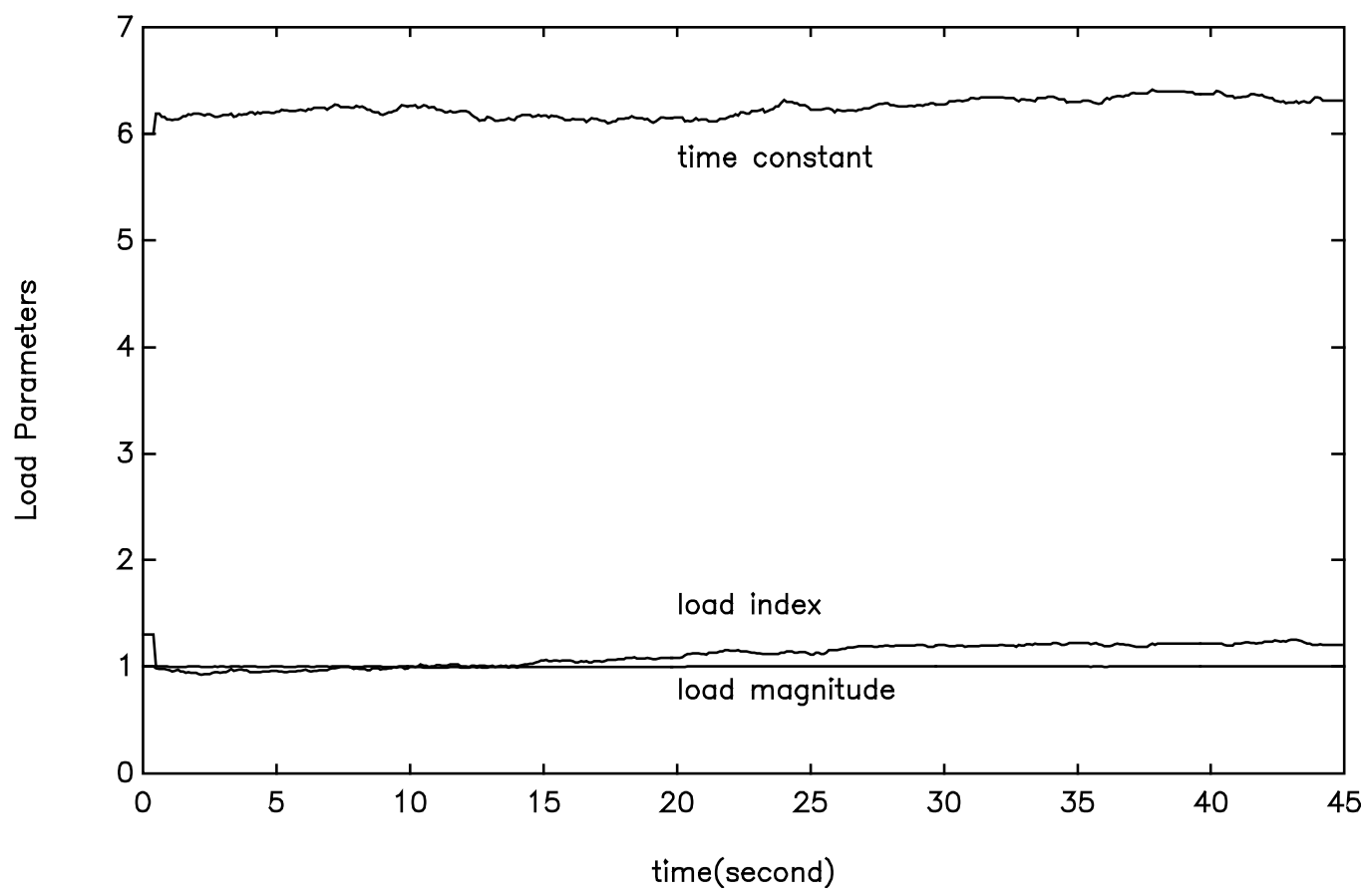

Figure 5a. Continuous estimation of load parameters with successive linearization method

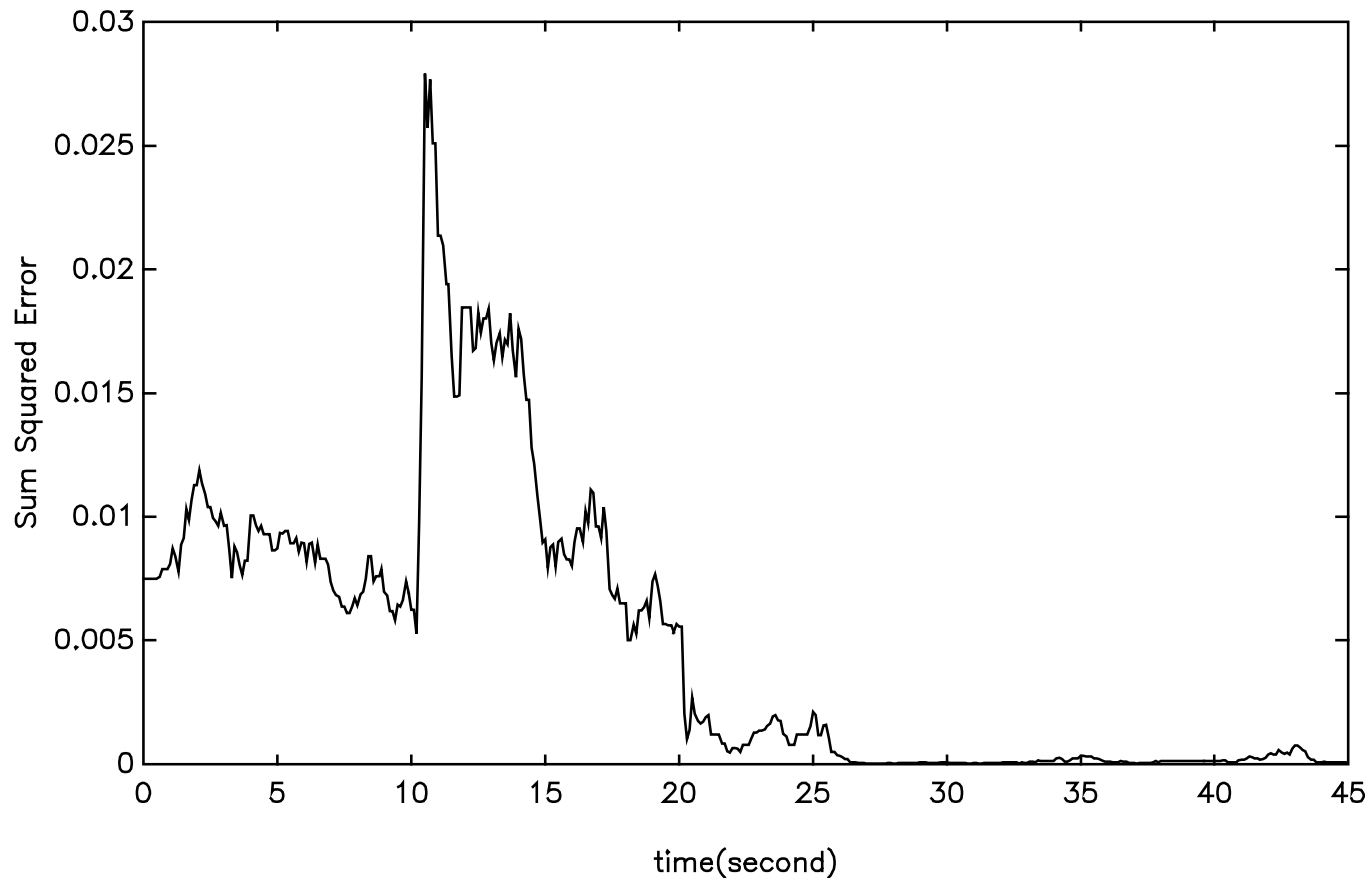

Figure 5b. Prediction sum squared error over the whole range data with load parameters identified from each sample window

The successive linearization approach achieves good trade-off among accuracy and robustness, and computing time. The algorithm needs very short training segment and acceptable computing time. All these merits make the successive linearization method very appropriate for continuously tracking variations of load characteristics.

It should be pointed out that real aggregate power demands may contain complicate load components thus demonstrate quite different characteristics. By investigating different categories of aggregate power demands, including residential, commercial, municipal/governmental and various industrial loads, typical load parameter sets of these load types could be obtained, which in turn can be served in different category load parameter identification. Besides, the proposed model is based on the successive linearization. During voltage transients caused by severe disturbances, the developed load may lead to significant errors. While the estimation errors can be exploited to correct the prediction so that the prediction error gradually converges to an acceptable level, some other measures could also be adopted to improve 
the performance. One method under investigation is to study aggregate power demands within a variety of load voltage ranges. Different sets of training segments are then sampled to acquire a number of initial parameter sets that could be applied at points when large voltage disturbances occur.

\subsection{Voltage Regulation}

The six-bus Ward-Hale system [12] is used to verify the importance of considering dynamic responses of aggregate power demands in voltage control. The test system contains the two LTC (load tap changing) transformer controlled voltages $\mathrm{V}_{1}$ and $\mathrm{V}_{2}$ on Bus-1 and Bus-2 (Fig.6). With the developed dynamic load model, power loads on both nodes are assumed to be constant $\mathrm{P}$ and $\mathrm{Q}$ with time constants of 15 $\mathrm{s}$ (mid-term voltage stability is concerned). Due to an initial reactive power demands increase, voltages at Bus-1 and Bus-2 are lower than the nominal system voltage. By identifying the load parameters and perform the LTP control accordingly (with variable duty cycle), the load voltages are controlled to the targets smoothly. By using conventional voltage regulation method, the LTC control is performed with a fixed duty cycle, which leads to excessive transients although $\mathrm{V}_{1}$ and $\mathrm{V}_{2}$ are also controlled to the targets finally (Fig. 7).

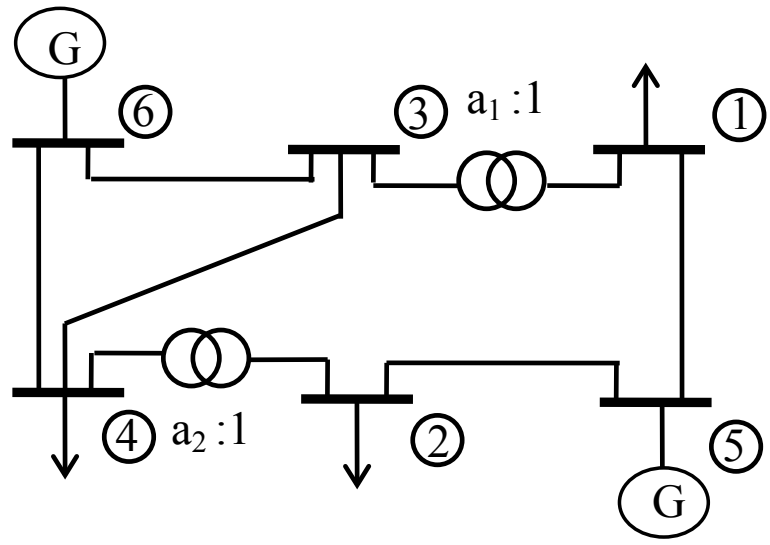

Figure 6. Multi-LTC Ward-Hale System

Without due respect to the dynamic characteristics of power demands (and some other factors), voltage control may cause reverse actions. Under a more severe disturbance, fixed duty cycle LTP control drives the power system into voltage collapse (Fig. 8a), which is due to excessively hunting for voltage variations. On the contrary, with the load parameters being incorporated into the voltage control, the LTP control is performed with an adaptive duty cycle so that $\mathrm{V}_{1}$ and $\mathrm{V}_{2}$ can converge achieved at the target voltages finally (Fig. 8b).

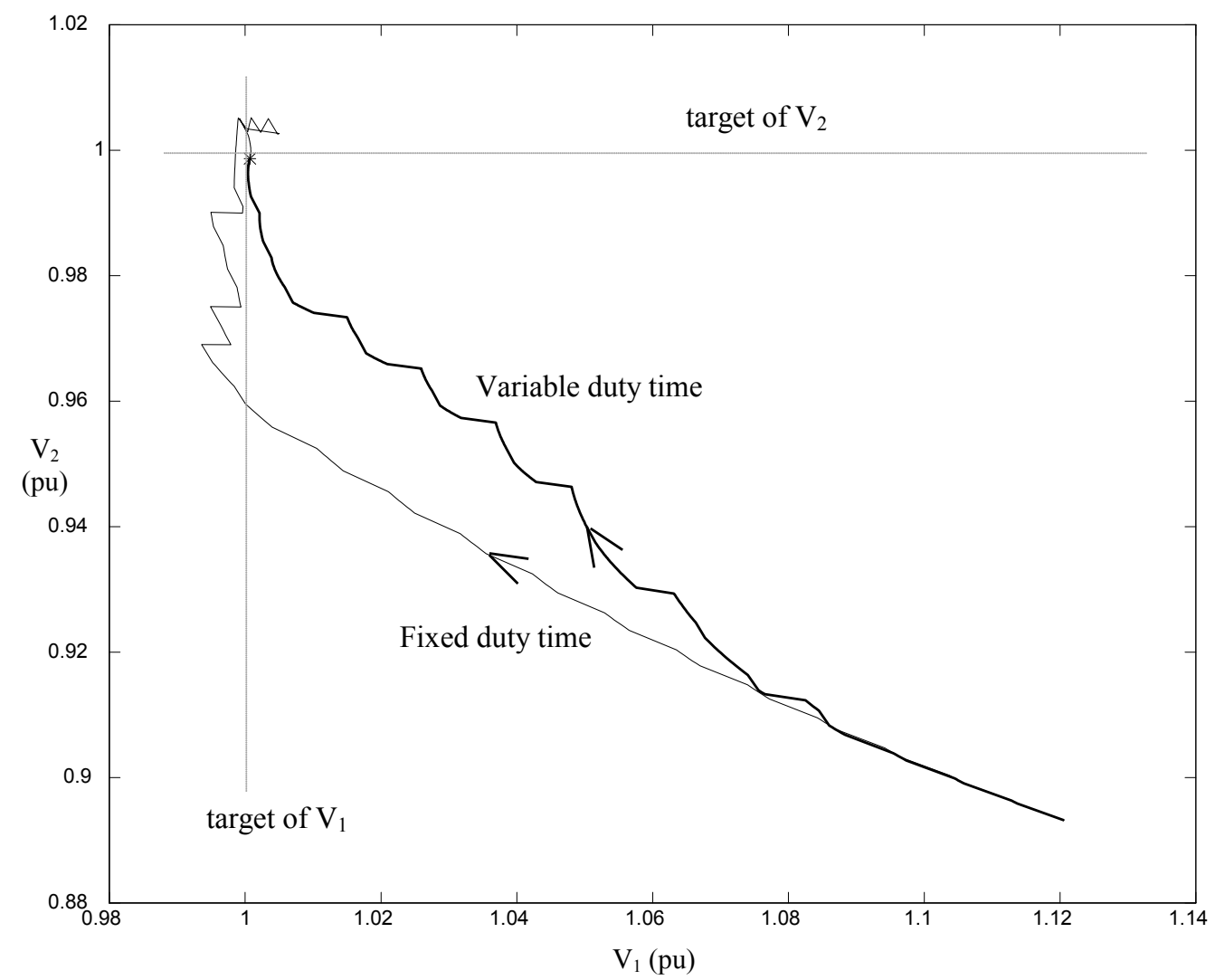

Figure 7. LTC Performance for Small Reactive Demand Increase 


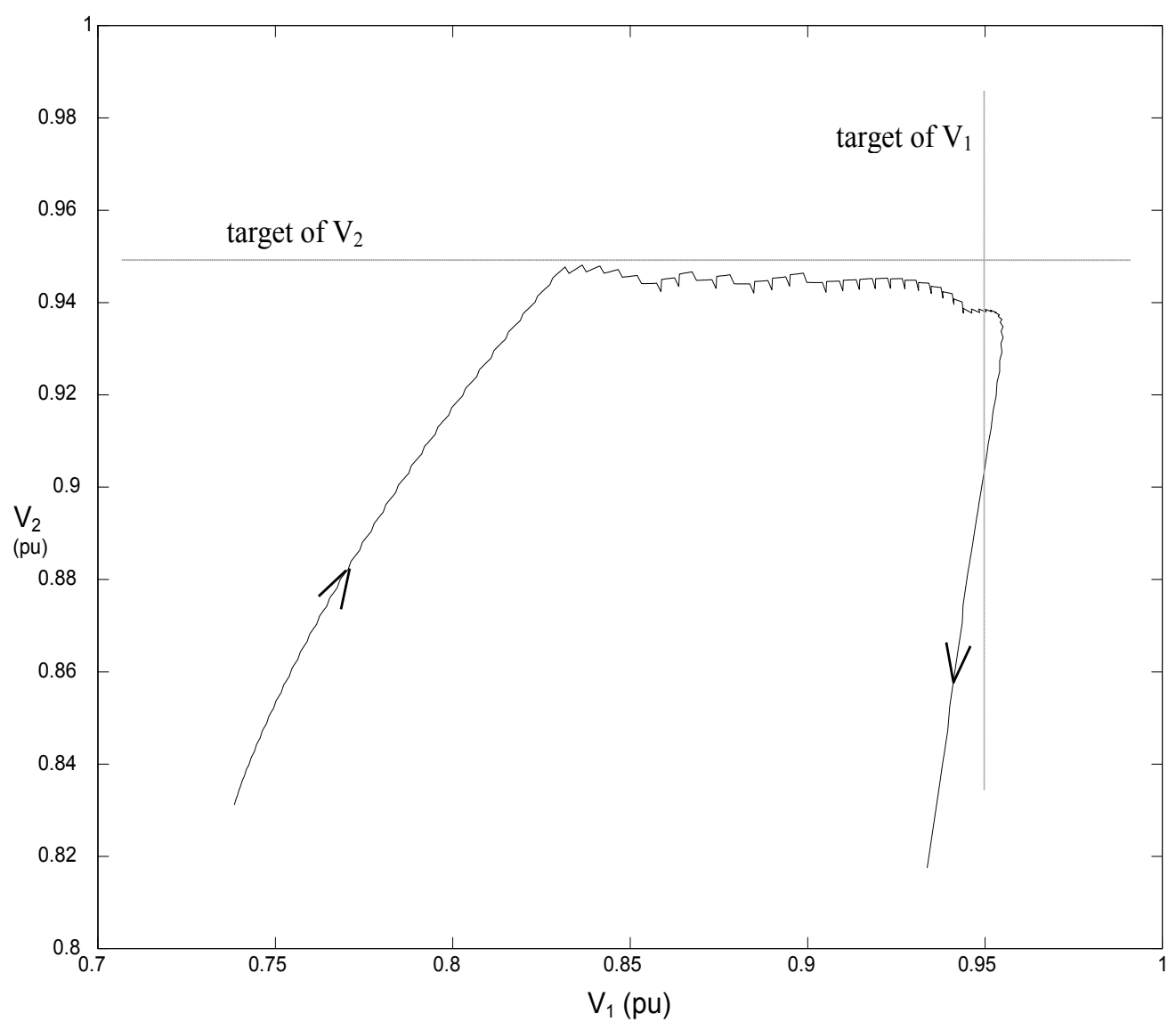

Figure 8a. LTC performance for large reactive power demand increase without considering dynamic load characteristics (fixed duty cycle)

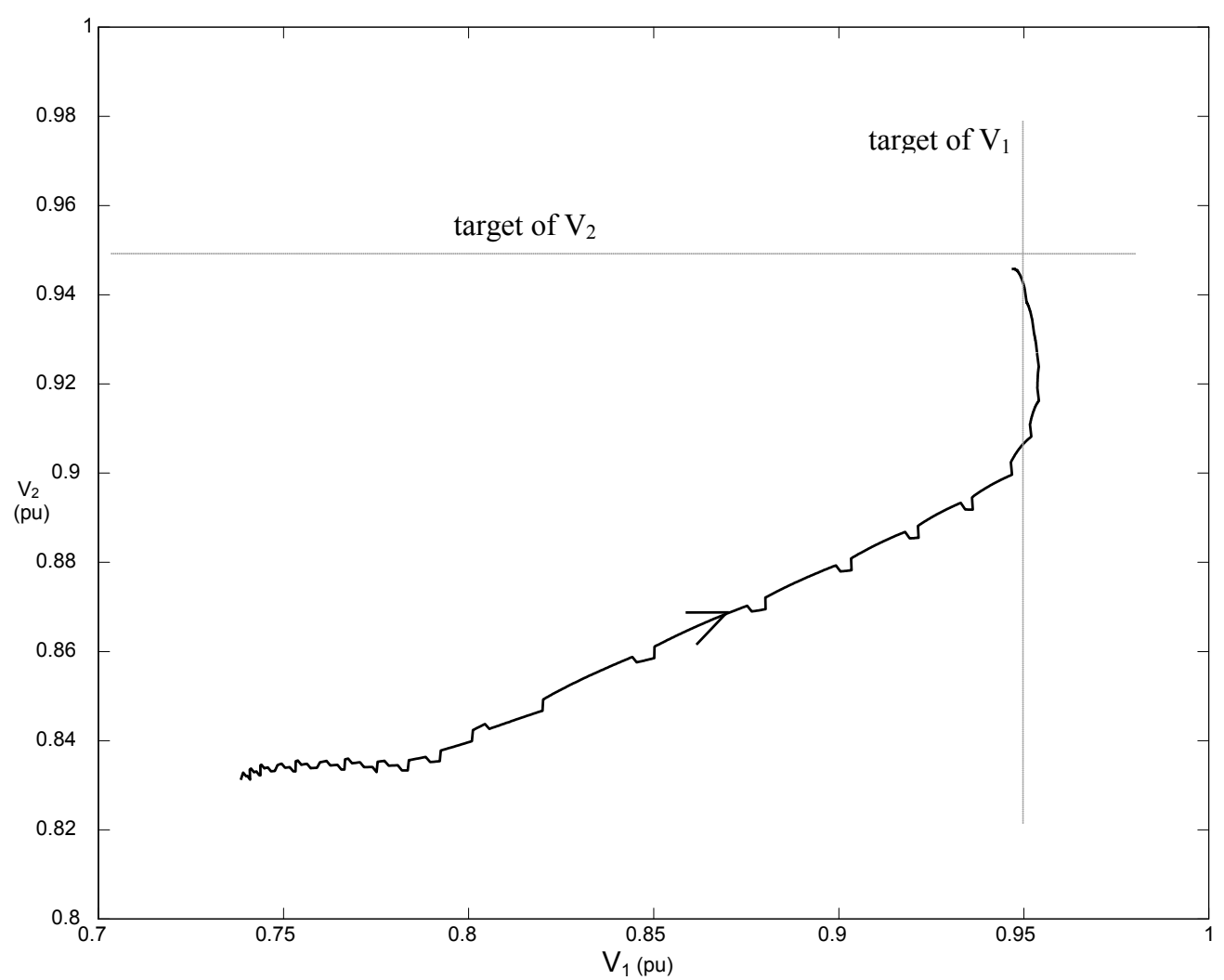

Figure 8b. LTC performance for large reactive power demand increase with considering dynamic load characteristics (variable duty cycle) 


\section{Conclusions}

With continuous voltage curve represented by a set of step changes, this paper proposes a discrete load model for fast evaluation of the dynamic response of consumer loads. The load model is constructed with load parameters that are often adopted to describe consumer characteristics. A test system with an induction motor and a resistor is used to assess the performance of the fast load modelling. An identification algorithm is proposed to evaluate parameters of the test system. By comparing results with those generated from detailed simulation, it is shown that the fast load model gives an accurate evaluation of the consumer demand under both steady state and transient conditions. The simulation shows that, by taking into account the dynamic characteristics of aggregate power demands in voltage regulation, intensive transients and reverse actions can be effectively suppressed.

The proposed discrete load model needs further verification through case studies using real site measurements, which has been planned in our future research.

\section{REFERENCES}

[1] J. S. Huang, "An Intelligent Control Scheme of Load-Tap-Changing Transforms for Voltage Stability Enhancement", International Conference on Artificial Intelligence in Science and Technology, AISAT 2004, Hobart, Australia, November 21-25, 2004.

[2] C. S. Chang and J. S. Huang, "Worst-Case Identification of Reactive Power Margin and Local Weakness of Power Systems", Electric Power Systems Research, Vol. 44, No. 2, February 1998, pp $77-83$

[3] Zheng Xiaoyu, Ma Jin, He Renmu, and Tang Yonghong, "Classification and Generalization of the Load Model Based on Model Dynamic Responses", Transactions China Electrotechnical Society, 2009-02.

[4] YANG Hua-chun, HE Ren-mu, WANG Peng, SHI Jing-hai and ZHANG Jin, "Load-modeling in Large-scale Grid Based on the Aggregation Theory", Automation of Electric Power Systems, 2005-01.

[5] G. Shackshaft, O.C. Symons and J.G. Hadwick, "General purpose model of power system loads", Proceedings of IEE, Vol. 124, No. 8, pp 715-723, August 1977.

[6] David J. Hill, "Nonlinear dynamic load models with recovery for voltage stability studies", IEEE Transactions on Power System, Vol. 8, No. 1, pp 166-176, Feb. 1993

[7] Daniel Karisson and David J. Hill, "Modeling and identification of nonlinear dynamic loads in power systems", IEEE Transactions on Power Systems, Vol. 9, No. 1, pp 157-166, February, 1994.

[8] T. Ohyama, A. Watanabe, K. Nishimura, and S. Tsuruta, "Voltage dependence of composite loads in power systems", IEEE Transactions on Power Apparatus and System, Vol. PAS-104, No. 11, pp 3064-3073, Nov. 1985

[9] Wilsun Xu and Y. Mansour, "Voltage stability analysis using generic dynamic load models", IEEE Transactions on Power Systems, Vol. 9, No. 1, pp 479-493, February, 1994.

[10] D. Karlsson, K. Linden, I. Segerqvist, and B. Stenborg, "Temporary load-voltage characteristics for voltage stability studies: field and laboratory measurements", CIGRE 1992 session, Paper 38-204, Paris, 30 August - 5 September, 1992.

[11] R.E. Kalaba, F.E. Udwadia, "Associative Memory Approach to the Identification of Structural and Mechanical System", Journal of Optimization Theory and Applications, Vol. 76, No. 2, pp 207-223, February, 1993.

[12] TC Calculator Help: 6 Bus System http://www.pserc.cornell.e $\mathrm{du} / \mathrm{tcc} / \mathrm{tcc}$ help.md?helpfile $=6$ bus.mc $\&$ windowtitle $=6 \% 20 \mathrm{Bu}$ s\%20System

[13] T. Kohonen, "Self-Organization and Associative Memory", Springer-Verlag, 1989, 119-157 